The MIT Laboratory for Nuclear Science (LNS)

Progress Report to the

U.S. Department of Energy

\title{
Design and Construction of Prototype Dark Matter Detectors
}

\section{Lepton Quark Studies (LQS)}

High Energy Physics Program 


\section{OVERVIEW}

The Lepton Quark Studies (LQS) group is engaged in searching for dark matter using the Dark Matter Time Projection Chamber (DMTPC) at the Waste Isolation Pilot Plant (WIPP) (Carlsbad, NM).

DMTPC is a direction-sensitive dark matter detector designed to measure the recoil direction and energy deposited by fluorine nuclei recoiling from the interaction with incident WIMPs. In the past year, the major areas of progress have been

- to publish the first dark matter search results from a surface run of the DMTPC prototype detector,

- to build and install the 10L prototype in the underground laboratory at WIPP which will house the $1 \mathrm{~m}^{3}$ detector, and

- to demonstrate charge and PMT readout of the TPC using prototype detectors, which allow triggering and $\Delta z$ measurement to be used in the $1 \mathrm{~m}^{3}$ detector under development.

These milestones are briefly summarized in the following. Publications, theses, and talks given by collaboration members are listed in Section VI.

The collaboration operated a 10L DMTPC prototype detector continuously in a surface lab, in "WIMP-trigger" mode. The goal of this run was to study detector backgrounds to a WIMP search, as well as to develop the full data analysis software chain and calibration procedures prior to underground operations. We acquired a data set consisting of 167 runs of 1000 events of 5 second CCD exposures, collected without trigger or camera shutter. The typical up-time during this surface run was $80 \%$. After correcting the live time for spark rejection cuts, the surface run livetime is 11.96 days, for an exposure of 38.6 gm-days.

With ${ }^{252} \mathrm{Cf}$ and alpha calibration data we determined that the nuclear recoil energy threshold is $50 \mathrm{keV}$, and the energy and recoil angle resolution are $15 \%$ at $50 \mathrm{keV}$, and $20^{\circ}$ averaged over the ${ }^{252} \mathrm{Cf}$ neutron-induced recoil spectrum, respectively, from Monte Carlo simulation. We developed background rejection cuts, and achieved a rate of $5 \times 10^{-5} \mathrm{~Hz}$. The events remaining after all background cuts are consistent in rate and spectrum with the expected neutron-induced background at the surface. We published a 90\% C.L. upper limit 
on the spin-dependent WIMP-proton cross section of $2.0 \times 10^{-33} \mathrm{~cm}^{2}$ for $115 \mathrm{GeV} / \mathrm{c}^{2}$ dark matter particle mass using this data set, shown in Figure 1.

Over the past year, the DMTPC collaboration has installed and commissionioned a new underground laboratory at the Waste Isolation Pilot Plant (WIPP) near Carlsbad, NM. The underground laboratory site is 2150 feet underground, in the Permian salt deposit. This geological enviroment is low in radon and uranium- and thorium-decay backgrounds, and provides $\sim 10^{4}$ attenuation of the cosmic ray muon and muon-induced neutron background flux relative to the surface.

The laboratory is built inside a 30' x 10' shipping container (connex). The connex was outfitted with internal electrical wiring and lighting in Spring 2010, transported underground in Summer 2010, and the first DMTPC detectors were installed in August and October 2010 (these were a neutron veto prototype and the 10L detector described above, respectively). The connex is divided into three separate areas for cleanliness; in the cleanest area, the particle counts are equivalent to surface laboratories at MIT. A photograph of the underground labpratory is shown in Figure 2.

The laboratory is configured to house the $1 \mathrm{~m}^{3}$ detector. The electrical configuration has 14120 VAC 20 Amp duplex receptacles on eight separate breakers, and UPS support for 4500 Watts. Environmental monitoring includes temperature sensors, particle counts, oxygen level, humidity sensor, and optical camera coverage of the underground laboratory. The temperature inside the laboratory is maintained by a 10,000 BTU air conditioner. The connex has optical fiber network connectivity to the surface, using two switches, currently configured for 32 IP addresses (this can be expanded in the future if necessary).

CCD readout of scintillation light produced in the mesh amplification region images low-energy nuclear recoil tracks with $\sim 0.5 \mathrm{~mm}$ position and length precision in the plane perpendicular to the drift direction. To measure recoil track properties in the drift direction, we have instrumented the mesh (and anode) planes with fast (integrating) amplifiers to read out the induced charge signal from the amplification region, on the R\&D prototype detector. The rise time of the fast amplifier signal on the mesh plane is well-correlated with the track extent in the direction parallel to the drift electric field. We have demonstrated comparable resolution to the CCD readout, using $100 \mathrm{keV}$ calibration tracks at known position and angle. This is shown in Figure 3. This charge signal readout will improve both the direction reconstruction resolution, and the background rejection capability of the $1 \mathrm{~m}^{3}$ DMTPC 


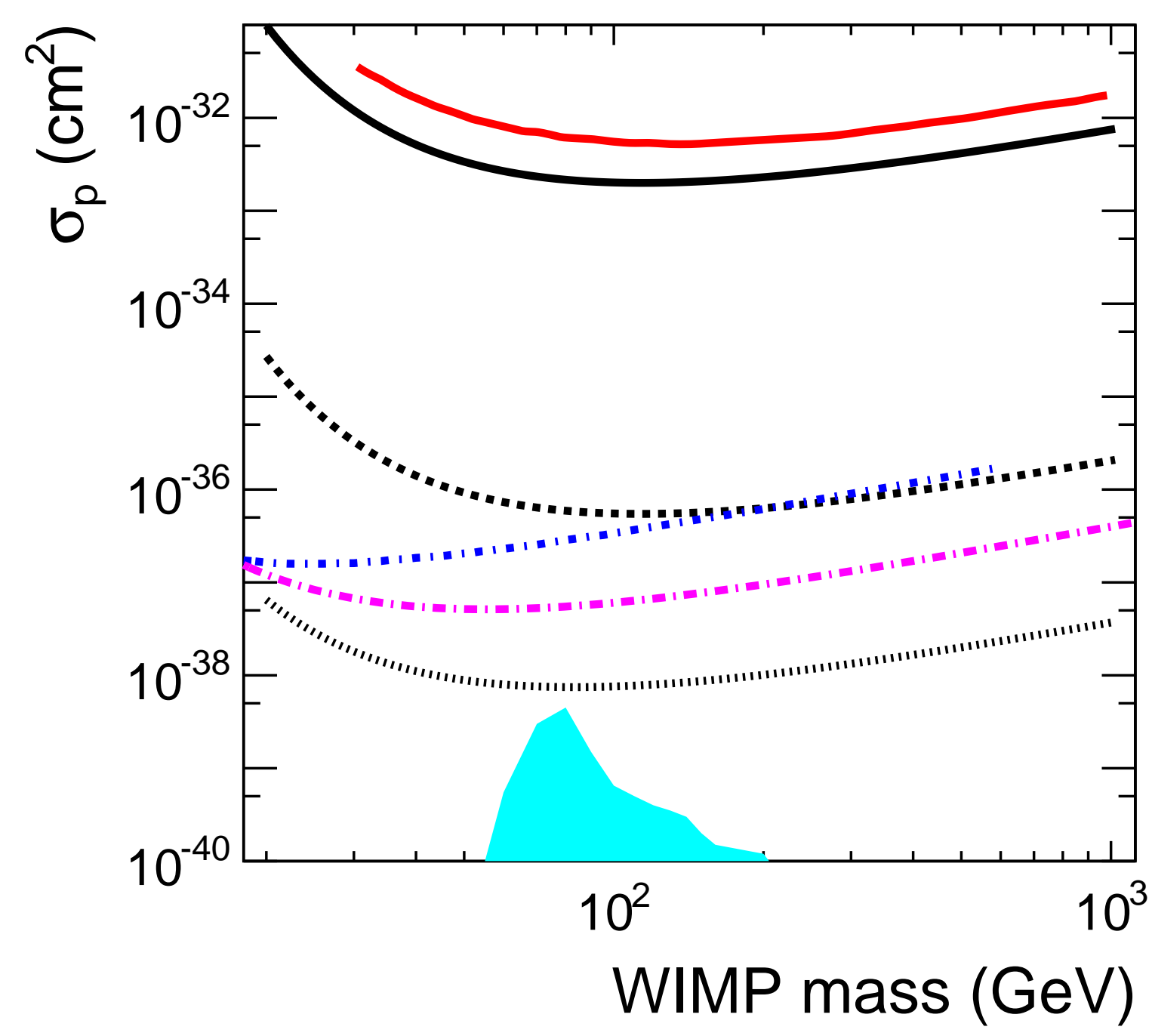

FIG. 1: Left: 90\% confidence level limit on the spin-dependent WIMP-proton cross section vs. dark matter particle mass from DMTPC surface data (black solid line), compared with the NEWAGE underground directional result (red solid line), and the two leading limits from conventional detectors, COUPP (magenta dash-dotted line) and PICASSO (blue dash-dotted line). The cyan shaded region shows MSSM parameter space. The projected sensitivity for DMTPC at WIPP, with 1 year exposure (black dashed line), and a $1 \mathrm{~m}^{3}$ detector at WIPP with $50 \mathrm{keV}$ energy threshold (black dotted line), are also shown. 


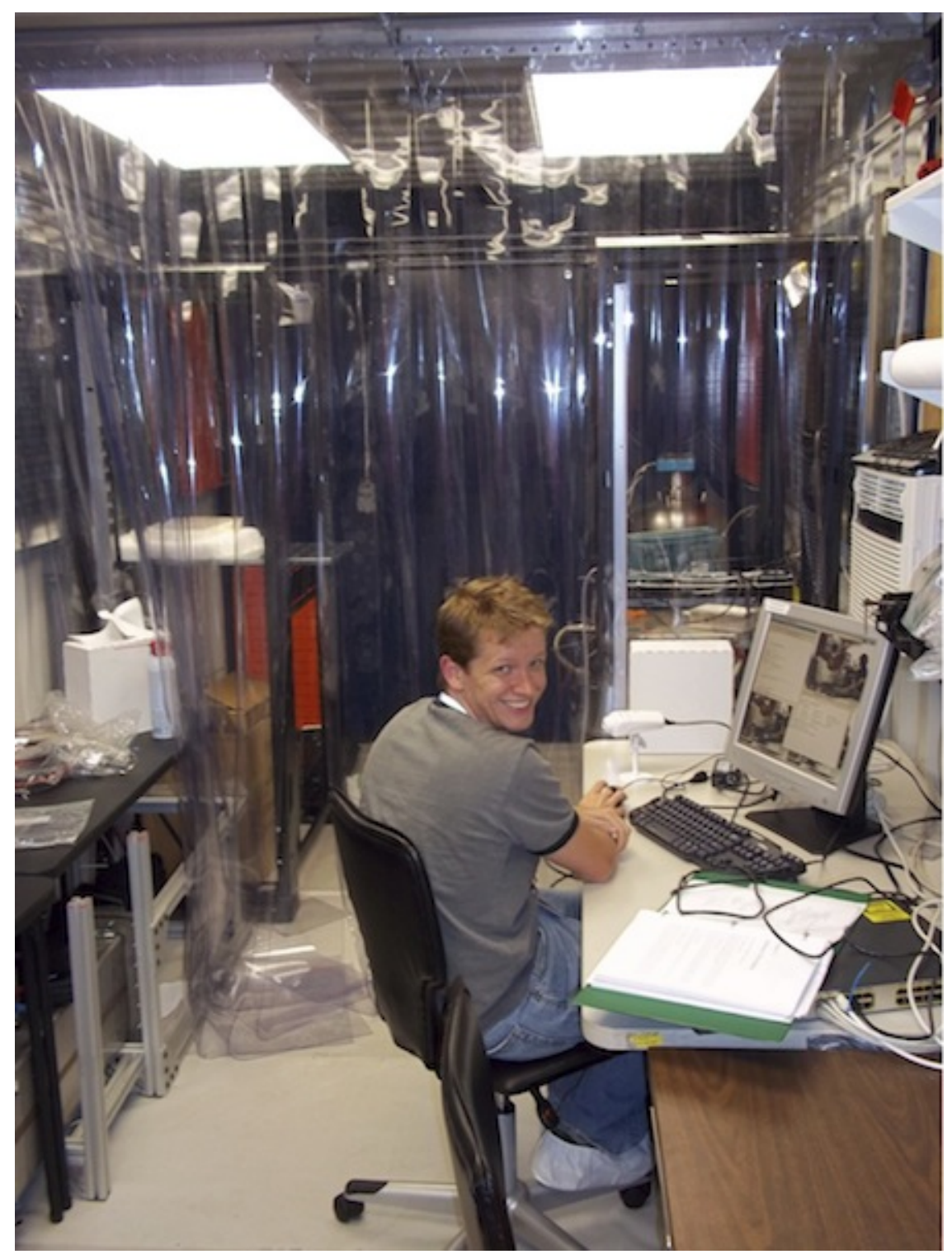

FIG. 2: DMTPC Underground Laboratory at the Waste Isolation Pilot Plant in Carlsbad, NM. The 10L DMTPC detector prototype is in the back right corner of the picture.

detector.

We have also instrumented the Cylon prototype detector with fast PMT readout, for the same

\section{PUBLICATIONS, THESES, AND CONFERENCE TALKS}

Publications in the past year:

[4] 'DMTPC: Dark matter detection with directional sensitivity," J.B.R. Battat, et al., 7 


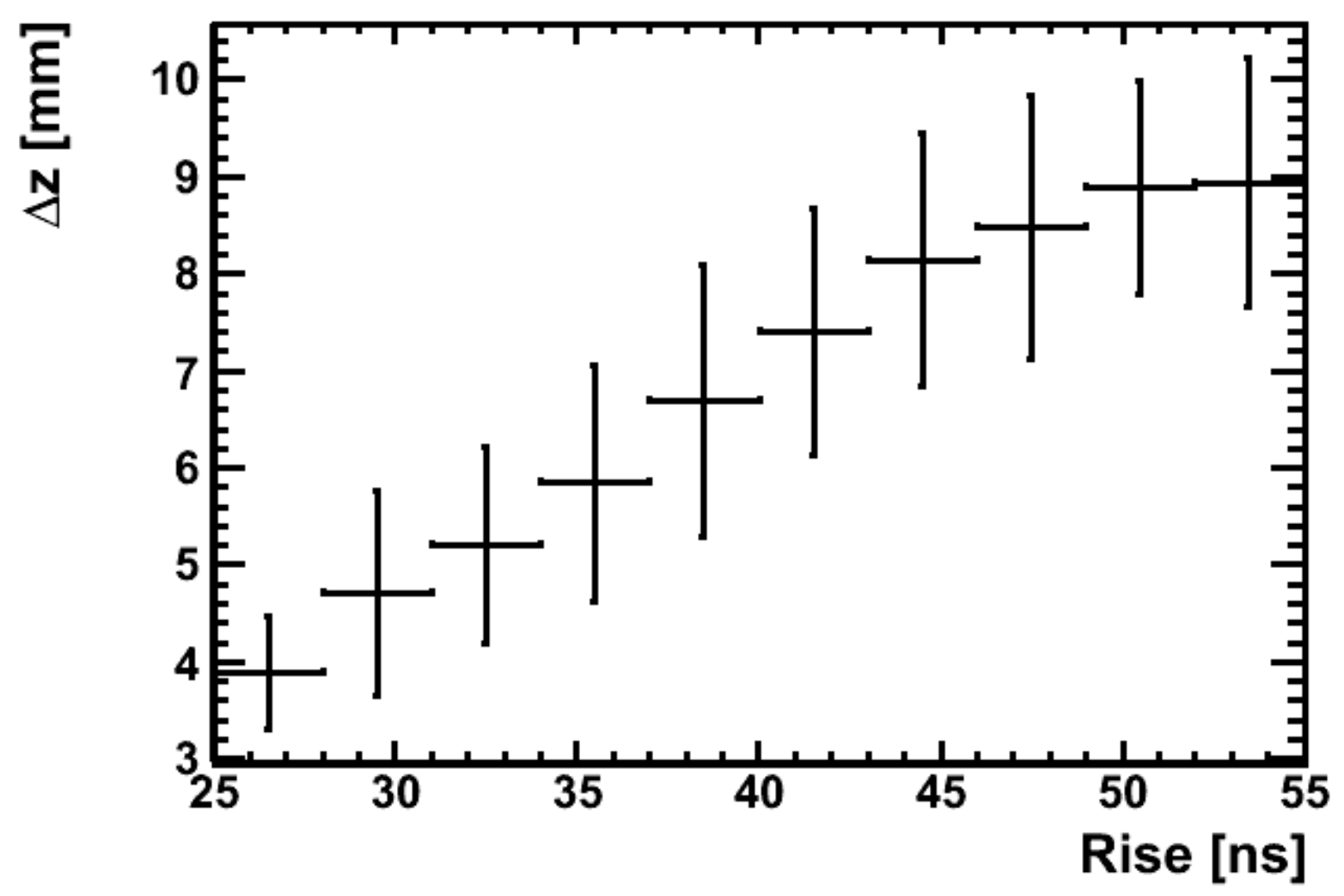

FIG. 3: Inferred track length in the drift direction $(\Delta z)$ vs. measured rise time, from a fast amplifier on the ground mesh plane.

pp, e-Print: arXiv:1012.3912 [astro-ph.IM].

[4] 'First Dark Matter Search Results from a Surface Run of the 10-L DMTPC Directional Dark Matter Detector," S. Ahlen et al., Phys.Lett. B695 (2011) 124-129, e-Print: arXiv:1006.2928 [hep-ex], Cited by 9 records

[4] 'Results from DMTPC 10-liter detector," D. Dujmic et al., 3 pp., Phys.Conf.Ser. 203 (2010) 012029

[4] 'The case for a directional dark matter detector and the status of current experimental efforts," S. Ahlenet al., 48 pp., Int.J.Mod.Phys. A25 (2010) 1-51, e-Print: arXiv:0911.0323 [astro-ph.CO], Cited by 31 records

[5] . P. Lees et al. [ The BABAR Collaboration ], "Cross Sections for the Reactions 
$e^{+} e^{-} \rightarrow K^{+} K^{-} \pi^{+} \pi^{-}, K^{+} K^{-} \pi^{0} \pi^{0}$, and $K^{+} K^{-} K^{+} K^{-}$Measured Using Initial-State Radiation," [arXiv:1103.3001 [hep-ex]].

[6] J. P. Lees et al. [ The BABAR Collaboration ], "Measurement of the mass and width of the Ds1(2536)+ meson," Phys. Rev. D83, 072003 (2011). [arXiv:1103.2675 [hep-ex]].

[7] P. del Amo Sanchez et al. [ BABAR Collaboration ], "Searches for the baryon- and lepton-number violating decays $B^{0} \rightarrow \Lambda_{c}^{+} \ell^{-}, B^{-} \rightarrow \Lambda \ell^{-}$, and $B^{-} \rightarrow \bar{\Lambda} \ell^{-}$," Phys. Rev. D83, 091101 (2011). [arXiv:1101.3830 [hep-ex]].

[8] P. del Amo Sanchez et al. [ The BABAR Collaboration ], "Measurement of partial branching fractions of inclusive charmless B meson decays to $\mathrm{K}+$, K0, and pi+," Phys. Rev. D83, 031103 (2011). [arXiv:1012.5031 [hep-ex]].

[9] P. del Amo Sanchez et al. [ BABAR Collaboration ], "Measurements of branching fractions, polarizations, and direct $\mathrm{CP}$-violation asymmetries in $\mathrm{B}+-i$, rho0 $\mathrm{K}^{*}+$ and B+ - ¿ f0(980)K*+ decays," Phys. Rev. D83, 051101 (2011). [arXiv:1012.4044 [hep$\mathrm{ex}]]$.

[10] P. del Amo Sanchez et al. [ The BABAR Collaboration ], "Analysis of the $D^{+} \rightarrow$ $K^{-} \pi^{+} e^{+} \nu_{e}$ decay channel," Phys. Rev. D83, 072001 (2011). [arXiv:1012.1810 [hepex]].

[11] P. del Amo Sanchez et al. [ The BABAR Collaboration ], "Dalitz plot analysis of $D_{s}^{+} \rightarrow K^{+} K^{-} \pi^{+}, "$ Phys. Rev. D83, 052001 (2011). [arXiv:1011.4190 [hep-ex]].

[12] P. del Amo Sanchez et al. [ The BaBar Collaboration ], "Studies of tau- -i eta.K-.nu and tau- - $i$ eta.pi-.nu at BaBar and a search for a second-class current," Phys. Rev. D83, 032002 (2011). [arXiv:1011.3917 [hep-ex]].

[13] P. del Amo Sanchez et al. [ The BABAR Collaboration ], "Measurement of the B - i D-bar $(*) D(*) K$ branching fractions," Phys. Rev. D83, 032004 (2011). [arXiv:1011.3929 [hep-ex]].

[14] P. del Amo Sanchez et al. [ The BABAR Collaboration ], "Search for the Decay $B^{0} \rightarrow \gamma \gamma, "$ Phys. Rev. D83, 032006 (2011). [arXiv:1010.2229 [hep-ex]]. 
[15] P. del Amo Sanchez et al. [BABAR Collaboration ], "Measurement of the $B^{0} \rightarrow \pi^{\ell} \ell^{+} \nu$ and $B^{+} \rightarrow \eta^{\left({ }^{\prime}\right)} \ell^{+} \nu$ Branching Fractions, the $B^{0} \rightarrow \pi^{-} \ell^{+} \nu$ and $B^{+} \rightarrow \eta \ell^{+} \nu$ Form-Factor Shapes, and Determination of $\left|V_{u b}\right|$, , Phys. Rev. D83, 052011 (2011). [arXiv:1010.0987 [hep-ex]].

[16] P. del Amo Sanchez et al. [ BABAR Collaboration ], "Study of $B \rightarrow \pi \ell \nu$ and $B \rightarrow \rho \ell \nu$ Decays and Determination of $\left|V_{u b}\right|$," Phys. Rev. D83, 032007 (2011). [arXiv:1005.3288 [hep-ex]].

PhD theses in the past year:

- "Detector Development for Direction-Sensitive Dark Matter Research," Hidefumi Tomita (Boston University Department of Physics PhD thesis) 2011

Conference talks in the past year:

- Dr. James Battat, "Directional Detection of Dark Matter," 5th Symposium on Large TPCs for Low Energy Rare Event Detection, Paris, France (2010)

- Dr. James Battat, "DMTPC: a direction-sensitive dark matter detector - first limits," Identification of Dark Matter, Montpelier, France (2010)

- Dr. James Battat, "Directional Detection of Dark Matter with DMTPC," PCTS Workshop (2010)

- Dr. James Battat, "DMTPC: a Direction-Sensitive Dark Matter Detector" 2010 UCLA Conference on Dark Matter and Dark Energy

- Dr. James Battat, "DMTPC: a Direction-Sensitive Dark Matter Detector" 2010 April APS Meeting

- Shawn Henderson, "DMTPC: A Dark Matter Detector with Directional Sensitivity," TeVPA 2009

- Dr. Denis Dujmic, "Results from DMTPC 10-Liter Prototype Detector," TAUP 2009

- Asher Kaboth, "DMTPC," 5th Patras Workshop on Axions, WIMPs and WISPs 